\title{
Mitochondrial DNA copy number alterations in familial mediterranean fever patients
}

\author{
Erdem $\mathrm{HB}^{1,2}$, Ceylan $\mathrm{AC}^{3}$, Sahin $\mathrm{I}^{1,2}$, Sever-Erdem $\mathrm{Z}^{4}$, Citli $\mathrm{S}^{3}$, Tatar $\mathrm{A}^{1}$ \\ Department of Medical Genetics, Ataturk University Medical Faculty, Erzurum, Turkey. \\ haktanbagis@atauni.edu.tr.
}

\section{ABSTRACT}

OBJECTIVES AND BACKGROUND: Familial Mediterranean Fever (FMF) is characterized by recurrent fever episodes as a result of inflammation of serous membranes. Changes in the number of different mtDNA copy number variations, detected in FMF patients, who developed amyloidosis, might be an important parameter in the understanding of the pathophysiology of the disease.

METHODS: Changes in the mtDNA copy number between 50 patients with FMF, who had M694V homozygote mutation and amyloidosis, and 50 healthy controls, who had not any MEFV mutation or FMF clinical finding, were examined. The 22 MEFV mutations were analyzed by Pyromark Q24 system. Quantitative analysis was performed on RT-PCR. The level of mtDNA was calculated using the delta $\mathrm{Ct}(\Delta \mathrm{Ct})$ of average $\mathrm{Ct}$ of mtDNA and $n D N A(\triangle C t=C t$ mtDNA-Ct nDNA) in the same well as an exponent of $2(2 \Delta \mathrm{Ct})$.

RESULTS: A significant decrease in the amount of mtDNA was detected in FMF patients with M694V homozygous mutation carriers, who developed amyloidosis compared to the control group $(p<0.001)$.

CONCLUSION: In this study, mitochondrial dysfunction, which has been identified through changes in the mitochondrial genome in many diseases, was identified by showing that the copy number variations of mtDNA in leukocytes also decreased for FMF disease (Tab. 3, Fig. 1, Ref. 21). Text in PDF www.elis.sk. KEY WORDS: familial mediterranean fever, mitochondrial DNA, amyloidosis, MEFV.

\section{Introduction}

Familial Mediterranean Fever (FMF) is an autosomal recessive inherited disorder, which is characterized by recurrent fever episodes, abdominal pain, chest pain and arthritis as the result of inflammation of serous membranes (1). In the Middle East and Anatolian populations, where the disease originated, the carrier frequency is $1 / 6-1 / 8$ and FMF is more common in these regions than in the rest of the world (2). Community of consanguineous marriages also increase the prevalence of FMF in Middle East and Anatolian populations. According to the previous studies, the carrier frequency in Turkish population was $1 / 5$ and the prevalence in Turkish population was 1/1075 (3). Currently, there is a huge number of patients, who are indicated for renal hemodialysis due

'Department of Medical Genetics, Ataturk University Medical Faculty, Erzurum, Turkey, ${ }^{2}$ University of Health Sciences, Ankara Diskapi Yildirim Beyazit Training and Research Hospital, Medical Genetics Unit, Ankara, Turkey, ${ }^{3}$ University of Health Sciences, Trabzon Kanuni Training and Research Hospital, Medical Genetics Unit, Trabzon, Turkey, and ${ }^{4}$ University of Health Sciences, Ankara Diskapi Yildirim Beyazit Training and Research Hospital, Family Medicine Clinic, Ankara, Turkey

Address for correspondence: H.B. Erdem, MD, University of Health Sciences, Ankara Diskapi Yildirim Beyazit Training and Research Hospital, Medical Genetics Unit, Ankara, Turkey.

Phone: +90.5535925736

Acknowledgement: Funding for this study was provided by Ataturk University (project number 2016/049). to chronic renal failure as the result of amyloidosis, because of the delayed diagnosis of FMF and/or disability of the patient's use of colchicine or resistance to colchicine.

In 1997, the International FMF Consortium and the French FMF Consortium reported that mutations in MEFV (MEditerranean FeVer) gene producing marenostrin and pyrine proteins on the 16 p13.3 region of chromosome 16 are responsible for the FMF (4, 5). M694V, M680I (G > C), M694I, V726A and E148Q are the most common variants in Anatolia, accounted for $74 \%$ of FMF patients $(6,7)$. Except for the M694V homozygous mutation, the effect of homozygosity / heterozygosity or compound heterozygosity of other mutations on the MEFV gene in terms of prognosis, has not been clearly determined yet. The mutation most associated with amyloidosis was shown to be the M694V homozygous mutation. Researchers still discuss whether the particularly common E148Q, V726A and R202Q variants are disease-related and should be considered as polymorphism (8-10).

Human mitochondrial DNA (mtDNA) has a double-stranded and circular structure. It contains protein coding and non-coding regions and is composed of about 16,569 nucleotides. There are 37 genes, 2 rRNA, 22 tRNA and 13 mRNA in mtDNA and there is a large number of copies of mtDNA that increase independently in the cell (11). The number of copies can vary from 100 to 100,000 depending on the cell type (12).

Although the pathology of FMF is linked to genetic and biochemical factors, the disease mechanism has not been fully elucidated. In the pathogenesis of some rheumatic diseases, the effect 
Tab. 1. Gender and age characteristics of the patient and control group.

\begin{tabular}{llcc}
\hline Group & $\mathrm{N}$ & Gender & Age \\
\hline FMF & 50 & Male(32)/Female(18) & $33.4 \pm 5.6$ \\
Control & 50 & Male(30)/Female(20) & $30.8 \pm 4.2$ \\
\hline
\end{tabular}

Tab. 2. The sequences of primers of ND-1 and HBB genes.

\begin{tabular}{ll}
\hline Gene & \multicolumn{1}{c}{ Sequence of primer } \\
\hline ND-1/mtDNA (Forward) & TTAGTTGCTTGGTTGTGTATTCC \\
ND-1/mtDNA (Reverse) & GAAAAAGGTAAAAAACTCTTTCAAGC \\
\hline HBB (Forward) & GGAGATGCCTCAGAAACTGC \\
HBB (Reverse) & AGGTTGGAGGTCGGAAAGTT \\
\hline
\end{tabular}

of insufficient ATP due to mitochondrial dysfunction was shown in the previous studies (13). The genetic alterations in mtDNA are the most important cause of mitochondrial dysfunction. One of these changes, mtDNA copy number variations, was shown to cause mitochondrial dysfunction in many diseases (14-16). There may also be a pathophysiological basis for the mitochondrial dysfunction with changes in the number of mtDNA copies in FMF.

FMF is a disease with limited biomarkers and amyloidosis that is a frequent and important complication, such as chronic renal failure. For this reason, it is considered that the changes in the number of different mtDNA copy number variations, detected in FMF patients, who developed amyloidosis, are an important parameter in the understanding the pathophysiology of the disease.

\section{Materials and methods}

\section{Patient selection}

In our study, changes in the mtDNA copy number between 50 patients with FMF, who had M694V homozygote mutation in MEFV gene and amyloidosis, and 50 healthy controls, who did not have any MEFV mutation or FMF clinical finding, were examined. DNA samples, isolated from peripheral blood, were taken from individuals, consulted to Ataturk University Medical Faculty Medical Genetics Department. All of the individuals provided a written informed consent before participating in the study, which was approved by the Ethical Committee of the Ataturk University (decision number; 2016-4/6). The age range of the patient group and the control group was determined to be 18-40. Gender and age characteristics of the patient and control group are summarized in Table 1. Considering the effect of aging on mitochondrial dysfunction, in the selection of the control group, the mean age close to the average age of the patient group was provided. Individuals with autoinflammatory, chronic inflammatory and autoimmune diseases other than FMF were excluded from the study.

\section{Molecular analysis}

Blood samples were collected into EDTA tubes. DNA of the patients was extracted by QIAGEN®, EZ1 ${ }^{\circledR}$ Advanced XL (Veltek Associates, Inc.) system. The PCR reactions were performed in $50 \mu \mathrm{l}$ reaction mixture containing $18.5 \mu \mathrm{l}$ PCR master mix (Qiagen GmbH, Hilden Germany), $2 \mu$ l of each primer, $0.5 \mu 1$ Taq DNA polymerase, $17 \mu 1 \mathrm{H}_{2} \mathrm{O}$ and $10 \mu 1 \mathrm{mtDNA}$ in a thermal cycler (SensoQuest Labcycler, GmbH, Hilden, Germany) under the following conditions: $95{ }^{\circ} \mathrm{C}$ for $15 \mathrm{~min}$ (initial denaturation) followed by 32 cycles at $94{ }^{\circ} \mathrm{C}$ for $1 \mathrm{~min}$ (denaturation), $56^{\circ} \mathrm{C}$ for $1 \mathrm{~min}$ (annealing), $72{ }^{\circ} \mathrm{C}$ for $80 \mathrm{~s}$ (extension), and a final extension at $72{ }^{\circ} \mathrm{C}$ for $15 \mathrm{~min}$. The $22 \mathrm{MEFV}$ mutations, which are commonly seen in people living in Anatolia were analyzed by QIAGEN ${ }^{\circledR}$, Pyromark Q24 system with the specific primers. Results were evaluated with PyroMark Q24 Advanced Software, and M694V homozygous patients were selected for the study (Fig. 1). Quantitative analysis was performed on Rotor-Gene-Q (Heidelberg, Germany) RT-PCR to determine the mtDNA copy numbers of the samples. For the analysis, the regions selected from the nuclear DNA (nDNA) and mtDNA were taken as reference. The sequences of the primers, suitable for the NADH dehydrogenase-1 (ND-1) gene identified for the nDNA and the beta-globin
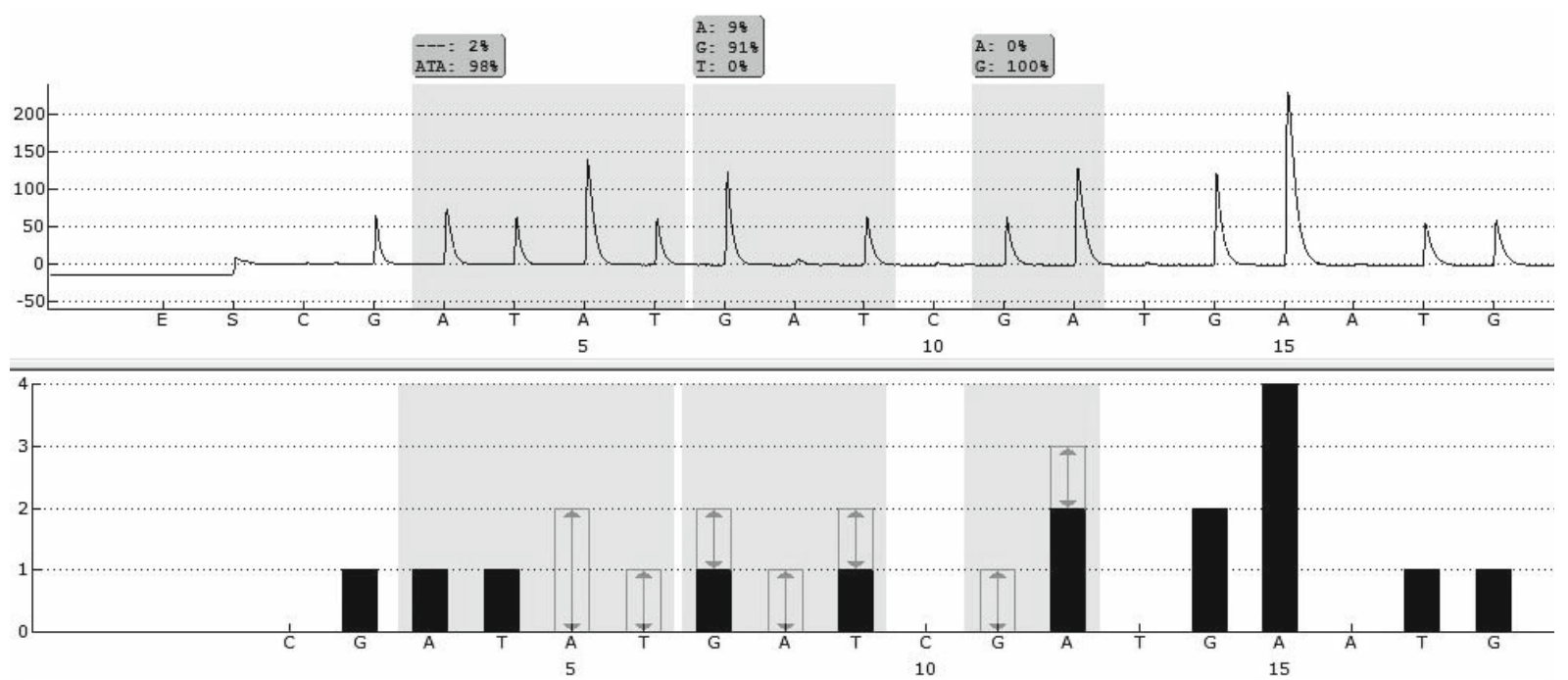

Fig. 1. Pyrosequence image of M694V homozygous FMF patient. 
Tab. 3. Statistical analysis of mtDNA copy numbers, detected in FMF patients and control group.

\begin{tabular}{lccccc}
\hline Group & $\mathrm{N}$ & Minimum & Maximum & Average & $\mathrm{p}$ \\
\hline FMF & 50 & 3,8637 & 119.4282 & 48.190998 & $<0.001$ \\
Control & 50 & 25,4572 & 171.2547 & 80.318280 & \\
\hline
\end{tabular}

(HBB) gene for mtDNA, are shown in Table 2. To determine the quantities of mtDNA and nDNA present in blood samples, the average threshold cycle number $(\mathrm{Ct})$ values of the nDNA and mtDNA were obtained from each case. The level of mtDNA was calculated using the delta $\mathrm{Ct}(\Delta \mathrm{Ct})$ of average $\mathrm{Ct}$ of mtDNA and nDNA $(\Delta \mathrm{Ct}=\mathrm{Ct}$ mtDNA-Ct nDNA $)$ in the same well as an exponent of $2\left(2^{\Delta \mathrm{Ct}}\right)$.

\section{Statistical analysis}

The data were evaluated in the SPSS Statistical Package program. Student T test was used to compare two independent groups in terms of numerical variables with normal distribution. Arithmetic mean, standard deviation, median, minimum and maximum values were given as descriptive statistics, $\mathrm{p}<0.05$ was considered statistically significant.

\section{Results}

As the result of analyses, the number of mtDNA copies in FMF patients and controls were found to be $48.19 \pm 27.3$ and $80.31 \pm$ 36.97 , respectively. There was also a significant difference in the statistical analysis, between the two groups $(\mathrm{p}<0.001)$ (Tab. 3).

\section{Discussion}

Mitochondria product almost all the ATP in the cell through oxidative phosphorylation. The most important disadvantage of the oxidative phosphorylation mechanism is that it is a source of reactive oxygen radicals. MtDNA does not have intron regions, histone proteins that provide protection from condensation and external influences, and sufficient DNA repair mechanisms. These conditions make mtDNA susceptible to oxidative damage (17).

Mitochondria are found in every cell type except erythrocytes, and even in the cells of different tissues belonging to the same organism, their number can change. For this reason, our study is based on the ratio of mtDNA to total DNA isolated, rather than the amount of mtDNA per mitochondria.

Previously, many diseases were associated with changes in mtDNA copy number. In relation to aging, it was also found that cognitive functions of women, who detected a high mtDNA in peripheral leukocytes due to increased oxidative stress were better than those that contained lower mtDNA copies in leukocytes (18). Brain tissue is unprotected against oxidative damage, so prolonged oxidative stress may increase dementia development (19). Considering the effect of age on the number of mtDNA copies, attention has been paid to the fact that the age and the average age of the patients and the control groups examined in our study are close to each other.
Mitochondrial dysfunction has also been associated with autism spectrum disorders (OSB), which are common behavioral disorders $(19,20)$. Unlike our study, there was an increase in mtDNA copy numbers in the studies related to OSB. This is explained by an increase in replication and a decrease in degradation as the result of the oxidative stress response (14). In the study about Huntington's disease, the number of mtDNA copies increased in patients, who had not yet started the disease clinically, but had a family history and had an increased CAG repeat in themselves; a decrease in the number of mtDNA copies in patients, who are in the onset of the clinical progression; whereas the number of mtDNA copies decreased in the advanced stages of the disease (21). In our study, patients were not classified according to the disease phase. Investigations of mtDNA copy numbers in patients with family history and M694V homozygote mutations, but do not have attacks yet; and the patients, who had attacks and clinical signs are thought to be able to provide insight into the changes in mtDNA copy number that can be seen in the life span of FMF patients.

MtDNA alterations have been investigated in some rheumatologic diseases, although there has been no previous study of mtDNA copy number or other changes in mtDNA in relation to FMF. In this context, the study with patients, who developed lupus nephritis followed by SLE showed a significant decrease in the number of mtDNA copies as in our study (13).

As conclusion, mtDNA copy number has the potential to be used as biomarker for many diseases and conditions. This study showed that FMF disease was inversely proportional to the number of mtDNA copies. In FMF patients, extended studies with different patient groups in relation to mtDNA copy number change are also important in order to clarify the molecular mechanism of the disease and to fully understand the effect of mitochondrial dysfunction on FMF.

\section{References}

1. Ben-Chetrit E, Levy M. Familial mediterranean fever. Lancet 1998; 351 (9103): 659-664.

2. Fujikura K. Global epidemiology of Familial Mediterranean fever mutations using population exome sequences. Mol Genet Genomic Med 2015; 3 (4): 272-282.

3. Yilmaz E, Ozen S, Balci B, Duzova A, Topaloglu R, Besbas $\mathbf{N}$ et al. Mutation frequency of familial Mediterranean fever and evidence for a high carrier rate in the Turkish population. Eur J Hum Genet 2001; 9 (7): 553-555.

4. Consortium FF. A candidate gene for familial Mediterranean fever. Nat Genet 1997; 17 (1): 25.

5. Consortium IF. Ancient missense mutations in a new member of the RoRet gene family are likely to cause familial Mediterranean fever. Cell 1997; 90 (4): 797-807.

6. Murat A, Anil AB, Ertaner D, Alkan B, YAVAŞCAN Ö, Nejat A et al. The Effect of M694V Mutation on Clinical Presentation and Acute Phase Response in Children with Familial Mediterranean Fever: Single Center Experience in Western Turkey. Turkiye Klinikleri J Med Sci 2011; 31 (6): 1410-1417. 


\section{5-428}

7. Soylemezoglu O, Kandur Y, Duzova A, Ozkaya O, Kasapcopur O, Baskin E et al. Familial Mediterranean fever with a single MEFV mutation: comparison of rare and common mutations in a Turkish paediatric cohort. Clin Exp Rheumatol 2014; 33 (6 Suppl 94): 152-155.

8. Coşku S, Kurtgöz S, Keskin E, Sönmez F, Bozkurt G. Frequency of mutations in Mediterranean fever gene, with gender and genotypephenotype correlations in a Turkish population. Journal Genet 2015; 94 (4): 629-635.

9. Habahbeh LA, al Hiary M, AI Zaben SF, Al-Momani A, Khasawneh R, abu Mallouh $M$ et al. Genetic profile of patients with familial Mediterranean fever (FMF): single center experience at King Hussein Medical Center (KHMC). Med Arch 2015; 69 (6): 417.

10. Giancane G, Ter Haar NM, Wulffraat N, Vastert SJ, Barron K, Hentgen $\mathbf{V}$ et al. Evidence-based recommendations for genetic diagnosis of familial Mediterranean fever. Ann Rheum Dis 2015; 74 (4): 635-641.

11. Smith A, Staden R, Young I. Sequence and organization of the human mitochondrial genome. Nature 1981; 290 (5806): 457-465.

12. Pikó L, Taylor KD. Amounts of mitochondrial DNA and abundance of some mitochondrial gene transcripts in early mouse embryos. Dev Biol $1987 ; 123$ (2): 364-374.

13. Lee H-T, Lin C-S, Chen W-S, Liao H-T, Tsai C-Y, Wei Y-H. Leukocyte mitochondrial DNA alteration in systemic lupus erythematosus and its relevance to the susceptibility to lupus nephritis. Int J Mol Sci 2012; 13 (7): 8853-8868.
14. Chen S, Li Z, He Y, Zhang F, Li H, Liao Y et al. Elevated mitochondrial DNA copy number in peripheral blood cells is associated with childhood autism. BMC psychiatry 2015; 15 (1): 1.

15. Liu CS, Kuo CL, Cheng WL, Huang CS, Lee CF, Wei YH. Alteration of the copy number of mitochondrial DNA in leukocytes of patients with hyperlipidemia. Ann N Y Acad Sci 2005; 1042 (1): 70-75.

16. Lemnrau A, Brook MN, Fletcher O, Coulson P, Tomczyk K, Jones $\mathbf{M}$ et al. Mitochondrial DNA copy number in peripheral blood cells and risk of developing breast cancer. Cancer Res 2015; 75 (14): 2844-2850.

17. Hosgood III HD, Liu C-S, Rothman N, Weinstein SJ, Bonner MR, Shen $\mathbf{M}$ et al. Mitochondrial DNA copy number and lung cancer risk in a prospective cohort study. Carcinogenesis 2010; 31 (5): 847-849.

18. Kim M-Y, Lee J-W, Kang H-C, Kim E, Lee D-C. Leukocyte mitochondrial DNA (mtDNA) content is associated with depression in old women. Arch Gerontol Geriatr 2011; 53 (2): e218-e221.

19. Barnham KJ, Masters CL, Bush AI. Neurodegenerative diseases and oxidative stress. Nat Rev Drug Discov 2004; 3 (3): 205.

20. Rossignol D, Frye R. Mitochondrial dysfunction in autism spectrum disorders: a systematic review and meta-analysis. Mol Psychiatry 2012; 17 (3): 290.

21. Petersen MH, Budtz-Jørgensen E, Sørensen SA, Nielsen JE, Hjermind LE, Vinther-Jensen T et al. Reduction in mitochondrial DNA copy number in peripheral leukocytes after onset of Huntington's disease. Mitochondrion 2014; 17: 14-21. 